\title{
LEVANTAMENTO DE LESÕES LABIAIS EM AGRICULTORES DO REMOTO INTERIOR PARAIBANO
}

\author{
MEASURING THE INCIDENCE OF LIP INJURIES IN FARMERS FROM \\ COUNTRYSIDE OF SERTÃO AREA OF PARAIBA
}

\author{
Kyara Dayse de Souza Pires ${ }^{1}$ \\ George João Ferreira do Nascimento ${ }^{2}$ \\ Letícia Pereira dos Santos ${ }^{3}$ \\ Rodolfo de Abreu Carolino ${ }^{4}$ \\ Gilmara Araújo de Sousa ${ }^{5}$ \\ Antônio Lopes Beserra Neto ${ }^{6}$ \\ Cyntia Helena Pereira de Carvalho ${ }^{7}$
}

RESUMO: Introdução: Pessoas que trabalham ao ar livre podem receber uma dose de radiação UV seis a oito vezes maiores em relação àquelas que trabalham em locais fechados. Consequentemente, estão mais sujeitas ao aparecimento de lesões dermatológicas e labiais, como carcinomas epidermóides e possivelmente outras formas de câncer de pele. Objetivo: Avaliar a prevalência de lesões labiais de uma população de agricultores no Sertão Paraibano, investigando ainda possíveis associações da presença das lesões com variáveis sociodemográficas e epidemiológicas. Metodologia: A coleta de dados deu-se por meio do exame clínico dos lábios e entrevista. A amostra foi de 97 agricultores, a maioria homens, feodermas de idade acima de 60 anos, de ensino fundamental incompleto e com renda até um salário mínimo. Os dados da pesquisa foram estruturados em banco de dados Microsolft Excel $2013^{\circledR}$ onde incialmente foi realizada uma análise descritiva das variáveis qualitativas e quantitativas. Para a análise estatística,

\footnotetext{
1 Graduada em Odontologia pela Universidade Federal de Campina Grande. kyaraodonto@gmail.com.br.

2 Professor Doutor do curso de Odontologia ligado à Universidade Federal de Campina Grande. geonascimento79@yahoo.com.br.

${ }^{3}$ Graduado em Odontologia pela Faculdade Santa Maria de Cajazeiras - PB. drleticia@outlook.com.

4 Graduado em Odontologia pela Universidade Federal de Campina Grande. rodolfoorg@yahoo.com.br.

5 Graduado em Odontologia pela Faculdade Santa Maria de Cajazeiras - PB. gilmaratsb@outlook.com.

6 Graduado em Odontologia pela Faculdade Santa Maria de Cajazeiras - PB. antoniolopesbeserraneto@gmail.com.

7 Professora Doutora do curso de Odontologia ligado à Universidade Federal de Campina Grande. cyntia_helena@yahoo.com.br.
} 
utilizou-se o software livre WinPepi for Windows 11.32. A associação entre as variáveis e a presença de lesões labiais foi verificada pelo teste Qui-quadrado e Teste de Mann-Whitney. Resultados: O boné foi a medida de proteção facial mais utilizada (70,9\%). Dos pesquisados, 63,9\% apresentavam lesão labial, sendo a queilite actínica a lesão mais encontrada com 42,5\%. A faixa etária apresentou significância estatística com a presença de lesão labial $(p=0.005)$, assim como a cor de pele $(p=0.0033)$, o tempo de exposição à radiação ultravioleta $(p=0.043)$ e 0 hábito de fumar $(p=0.0013)$. Conclusão: A prevalência das lesões labiais é alta na população estudada, que pode ser associada tanto aos fatores ambientais da região, bem como ao avançar de idade, grande exposição à radiação ultravioleta e ao fumo. Assim, é importante incentivar trabalhos que visem à educação em saúde para a prevenção de lesões labiais, em especial às potencialmente malignas.

Palavras-chave: Agricultor, Lábio, Radiação Solar.

ABSTRACT: Introduction: People who work outdoors can receive a dose of UV radiation six to eight times higher than those who work indoors. Consequently, they are more prone to the appearance of dermatological and labial lesions, such as squamous cell carcinomas and possibly other forms of skin cancer. Objective: Evaluating the prevalence of labral lesions in a population of farmers in the Sertão área of Paraíba also investigating possible associations of the presence of lesions with sociodemographic and epidemiological variables. Methodology: Data collection took place through clinical examination of the lips and interview. The sample consisted of 97 farmers, mostly men, feodermas aged over 60, with incomplete primary education and with income up to a minimum wage. The research data were structured in a Microsolft Excel 2013 database where initially a descriptive analysis of qualitative and quantitative variables was performed. For statistical analysis, the free software WinPepi for Windows 11.32 was used. The association between variables and the presence of lip lesions was verified by the Chi-square test and the MannWhitney test. Results: The cap was the most used facial protection measure (70.9\%). Of those surveyed, 63.9\% had a lip lesion, with actinic cheilitis as the most common lesion with $42.5 \%$. The age group showed statistical significance with the presence of lip lesion ( $p=0.005)$, as well as skin color ( $p=0.0033)$, the time of exposure to ultraviolet radiation $(p=0.043)$ and the smoking habit $(p=0.0013)$. Conclusion: The prevalence of lip lesions is high in the population studied, which can be associated with both the environmental factors of the region, as well as with advancing age, great exposure to ultraviolet radiation and smoking. Thus, it is important to encourage studies aimed at health education for the prevention of lip lesions, especially those that are potentially malignant.

Keywords: Farmer, Solar Radiation, Lip. 


\section{INTRODUÇÃO}

A radiação ultravioleta (UV) é um dos mais importantes fatores ambientais que afetam a saúde do ser humano até o momento (AUBIN, 2013), é responsável por diversos efeitos à saúde humana, benéficos como a síntese da vitamina $\mathrm{D}, \mathrm{e}$ maléficos como lesões malignas em pele (SILVA, 2008). Segundo o protocolo de Dermatoses Ocupacionais lançado em 2016 pelo Ministério de Sáude, as fotodermatoses, também denominadas fotodermatites ou lúcides, compreendem muitas reações anormais da pele causa0.das pela radiação UV ou pelo espectro visível da luz (BRASIL, 2016).

Os trabalhadores em várias atividades ocupacionais que se expõem à radiação UV por quatro ou mais horas nos horários de pico podem ser afetados, principalmente, se negligenciarem proteção adequada (BRASIL, 2016). Por esta razão, a exposição à radiação solar é uma questão de saúde pública mesmo que ainda sejam poucas as ações preventivas à exposição excessiva ao sol no Brasil (LUCENA et al, 2012)

Pessoas que trabalham ao ar livre podem receber uma dose de radiação UV seis a oito vezes maiores que as que trabalham em locais fechados (LUCENA et al, 2012). Consequentemente, estão mais sujeitas ao aparecimento de lesões dermatológicas e labiais, como carcinomas epidermóides e possivelmente outras formas de câncer de pele (DAVIS et al, 2002). Esse conhecimento epidemiológico não é usado suficientemente como ponto de partida para programas de prevenção no ambiente de trabalho em medicina ocupacional (LUCENA et al, 2012).

Mesmo diante do enorme volume de dados e estudos sobre lesões de pele e mucosa labial decorrentes da exposição solar, sabe-se que não está clara a patogênese dessas doenças. É consenso que o desenvolvimento dessas lesões não é um simples processo. A história natural dessas doenças é o resultado de vários fatores de risco internos e externos ao ser humano, atuando independentemente, antagonicamente ou sinergicamente para produzir alterações. Como resultado de 
longas, repetidas e intermitentes exposições a esses fatores de risco, lesões iniciais podem evoluir para neoplasias invasivas (LUCENA et al, 2012; CINTRA et al, 2013).

A exposição ocupacional à luz do sol tem sido relacionada com alguns tipos de doenças cutâneas e labiais, dentre as lesões labiais, as mais comuns são queilite actínica e efélides, mas também ocorrem outras lesões como carcinomas labial, melanoma, eritroplasia, herpes labial e leucoplasia (LUCENA et al, 2012; MARTINSFILHO, 2011).

De forma generalizada, as lesões labiais iniciais são assintomáticas e o aspecto clínico não evidencia a gravidade histopatológica da lesão (CINTRA et al, 2013). A terapêutica adotada para esta lesão é baseada em remoção cirúrgica de áreas ásperas do lábio acometido associada sempre a orientações e motivação do portador das lesões labiais sobre fotoproteção, sendo sempre de grande valia a avaliação histopatológica para o conhecimento dos graus de alterações naquele tecido (RIBEIRO, 2014).

Uma vez que não é possível separar os benefícios da luz solar de seus efeitos nocivos, é importante entender os riscos da superexposição e tomar simples precauções para sua proteção. Neste sentido, este estudo avaliou agricultores do remoto interior Paraibano que trabalham expostos a raios UV e, assim, fez um levantamento de lesões labiais nesta população, bem como, a conscientização destes indivíduos para a proteção solar.

\section{METODOLOGIA}

Este estudo é um estudo transversal do tipo observacional, realizado no interior da Paraíba, nas zonas rurais dos municípios de Patos, Cajazeiras e Sousa, sua amostra foi realizada por conveniência, que é justificada pela dificuldade de acesso à população estudada. Participaram desta pesquisa 97 agricultores que responderam a um questionário de (LICENA et al, 2012), contendo informações relativas a dados pessoais, informações de ocupação como: sexo, idade, cor de 
pele, escolaridade, renda, tempo de exposição solar (horas, dias e meses), tipo de fotoproteção utilizada na face, tabagismo e etilismo foram avaliados.

Foram realizados, então, exames clínicos dos lábios superiores e inferiores em sentido horário, por meio de manobras semiotécnicas de inspeção e palpação, com o objetivo de identificar lesões como quelite actínica, ceratose solar, efélides, melanoma, carcinoma labial, herpes labial, leucoplasia, eritroplasia, ente outras. Os examinadores estavam devidamente paramentados com os equipamentos de proteção individual, mascara, luva, gorro e óculos de proteção, e os exames eram realizados em áreas bem iluminadas e silenciosas sempre durante o dia.

Foram considerados no exame clínico: secura, atrofia, lesões escamosas, inchaço labial, eritema, ulcerações, demarcações turvas entre o vermelhão do lábio e pele, dobras demarcadas ao longo do lábio, manchas ou placas brancas, crostas, áreas manchadas ou com palidez. Câmeras fotográficas foram utilizadas para melhorar a visibilidade dos lábios por meio do recurso de aumento da imagem, bem como esclarecer possíveis dúvidas em relação ao diagnóstico.

O estudo incluiu indivíduos maiores de 18 anos de idade, que concordaram em participar da pesquisa através de assinatura do Termo de Consentimento Livre e Esclarecido. A pesquisa foi aprovada pelo Comitê de Ética em Pesquisa designado pela Plataforma Brasil com parecer 833.767 de 16/10/2014 (ANEXO A).

Os dados da pesquisa foram estruturados em banco de dados Microsolft Excel 2013 ® onde incialmente foi realizada uma análise descritiva das variáveis qualitativas e quantitativas. Para a análise estatística, utilizou-se o software livre WinPepi for Windows 11.32. A associação entre as variáveis e a presença de lesões labiais foi verificada pelo teste Qui-quadrado e Teste de Mann-Whitney. Para observar a magnitude dessas relações, utilizou-se intervalo de confiança de 95\%.

\section{RESULTADOS}

A amostra do trabalho foi de 97 agricultores, dos quais a maioria era do sexo masculino (60,8\%), feodermas (46,8\%), e idade acima de 60 anos com $41,9 \%$ da 
amostra. Em relação ao grau de escolaridade, a maioria apresentava ensino fundamental incompleto $(68,0 \%)$ e renda de até um salário mínimo (74,2\%).

Quando avaliado o tempo de exposição ocupacional em relação a horas por dia, 61,8\% trabalhavam expostos 4 a 8 horas diárias e aos dias da semana, 95\% dos agricultores trabalhavam mais que 5 dias semanais. $O$ boné ou chapéu foi o tipo de fotoproteção facial mais utilizado $(40,2 \%)$ e apenas $2,1 \%$ dos trabalhadores utilizavam filtro solar labial. O número de não-tabagistas e não-etilistas foi de $59,7 \%$ e $63,9 \%$, respectivamente.

Foi encontrada uma prevalência de lesões labiais em 62 agricultores, ou seja, uma prevalência de $64 \%$ do total da amostra. Quando relacionada a presença de lesão com as variáveis estudadas, não houve diferença significativa com o sexo ( $p=$ 0.107), mas com a faixa etária, encontrou-se uma associação significativa entre a idade o acometimento da lesão, onde os agricultores encaixados nas faixas etárias mais altas eram acometidos de lesão $(p=0.005)$. Além da faixa etária, foi possível observar que a cor de pele também apresentou relação estatística significante com a presença ou não de lesão no lábio dos agricultores, onde os agricultores feodermas têm menos risco de ser acometidos pelas lesões labiais estudadas ( $p=0.0033$ ). (Tabela 1).

Tabela 1 - Relação das variáveis do estudo com número de agricultores sem lesão e com lesão e valores de p. Patos/PB, 2016.

\begin{tabular}{l|c|c|c}
\hline \multicolumn{1}{c|}{ VARIÁVEIS } & $\begin{array}{c}\text { SEM LESÃO } \\
\mathbf{n}(\%)\end{array}$ & $\begin{array}{c}\text { COM LESÃO } \\
\mathbf{n}(\%)\end{array}$ & $\boldsymbol{p}$ \\
\hline $\begin{array}{l}\text { Sexo } \\
\text { Masculino }\end{array}$ & $24(69)$ & $35(57)$ & $0.107^{*}$ \\
Feminino & $11(31)$ & $27(43)$ & \\
\hline Faixa Etária & & & $\mathbf{0 . 0 0 5}(\mathrm{Z}=2.78)^{\star *}$ \\
$18-30$ anos & $04(11)$ & $02(03)$ & \\
31-45 anos & $11(31)$ & $13(21)$ & \\
$46-60$ anos & $10(29)$ & $21(34)$ & \\
$>$ 60 anos & $10(29)$ & $26(42)$ & \\
\hline Cor de pele & & & $\mathbf{0 . 0 0 3 3 ^ { * }}$ \\
Leucoderma & $07(20)$ & $17(27)$ & \\
Feoderma & $17(49)$ & $16(26)$ & \\
Melanoderma & $11(31)$ & $29(47)$ & \\
\hline Escolaridade & & $16(25)$ & \\
Analfabetos & $10(29)$ & &
\end{tabular}




\begin{tabular}{|c|c|c|c|}
\hline \multirow{6}{*}{$\begin{array}{l}\text { Ensino fundamental } \\
\text { incompleto } \\
\text { Ensino Fundamental completo } \\
\text { Ensino médio incompleto } \\
\text { Ensino médio completo } \\
\text { Ensino Superior } \\
\text { Pós-graduação }\end{array}$} & $24(69)$ & $42(64)$ & \\
\hline & - & $04(11)$ & \\
\hline & 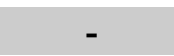 & - & \\
\hline & $01(2)$ & - & \\
\hline & - & - & \\
\hline & - & - & \\
\hline \multirow{4}{*}{$\begin{array}{l}\text { Renda } \\
\text { Menos de } \mathrm{R} \$ 788,00 \\
\mathrm{R} \$ 788,00 \text { a } \mathrm{R} \$ 1.500,00 \\
\text { Mais de } \mathrm{R} \$ 1.500,00\end{array}$} & & & $0.611(\mathrm{Z}=0.51)^{\star \star}$ \\
\hline & $24(71)$ & $47(70)$ & \\
\hline & $10(29)$ & $14(22)$ & \\
\hline & & $01(8)$ & \\
\hline Tempo de exposição (horas) & & & $<\underset{*}{0.0001(Z=4.89)^{*}}$ \\
\hline \multirow{3}{*}{$\begin{array}{l}\text { Menos de } 4 \text { horas/dia } \\
\text { De } 5 \text { a } 8 \text { horas/dia } \\
\text { Mais de } 8 \text { horas/dia }\end{array}$} & $23(66)$ & $11(18)$ & \\
\hline & $04(11)$ & $37(60)$ & \\
\hline & $08(23)$ & $14(22)$ & \\
\hline \multirow{5}{*}{$\begin{array}{l}\text { Tempo de exposição (dias) } \\
\text { Menos de } 5 \text { dias } \\
5 \text { dias } \\
6 \text { dias } \\
7 \text { dias }\end{array}$} & & & $0.043(\mathrm{Z}=2.02)^{\star *}$ \\
\hline & $05(14)$ & - & \\
\hline & $18(52)$ & $37(60)$ & \\
\hline & $07(20)$ & $14(22)$ & \\
\hline & $05(14)$ & $11(18)$ & \\
\hline \multirow{3}{*}{$\begin{array}{l}\text { Medida de Fotoproteção } \\
\text { Nenhuma } \\
\text { Alguma fotoproteção }\end{array}$} & & & $0.322^{*}$ \\
\hline & $16(46)$ & $36(54)$ & \\
\hline & 19(54) & $26(46)$ & \\
\hline \multirow{3}{*}{$\begin{array}{l}\text { Tabagismo } \\
\text { Fumantes } \\
\text { Não-fumantes }\end{array}$} & & & $0.013^{*}$ \\
\hline & $10(29)$ & $29(47)$ & \\
\hline & $25(71)$ & $33(53)$ & \\
\hline \multirow{4}{*}{$\begin{array}{l}\text { Etilismo } \\
\text { Etilistas } \\
\text { Não-etilistas } \\
\text { TOTAL }\end{array}$} & & & $0.299^{*}$ \\
\hline & $11(31)$ & $24(39)$ & \\
\hline & $24(69)$ & $38(61)$ & \\
\hline & $35(100)$ & $62(100)$ & $92(100)$ \\
\hline
\end{tabular}

Fonte: Do autor

* Teste de Qui-quadrado

** Teste de Mann-Whitney

Tabela 02 - Relação dos tipos de lesões labiais encontradas, número absoluto (n) e porcentagem (\%). Patos/PB, 2017.

\begin{tabular}{l|c|c}
\multicolumn{1}{c|}{ Tipo de lesão } & N & (\%) \\
\hline Queilite actínica & 31 & 42 \\
Efélides & 23 & 31 \\
Leucoplasia & 6 & 9 \\
Eritroplasia & 6 & 9 \\
Ceratose solar & 3 & 4 \\
Herpes & 3 & 4
\end{tabular}


Outras 1 1

Fonte: Do autor.

Em ralação à escolaridade e à renda, como já foi relatado, a maioria dos agricultores está enquadrada na baixa escolaridade e com renda até um salário mínimo, e, dessa forma, não foi possível encontrar relação entre essas variáveis e a presença ou não de lesão. (Tabela 1).

Ao relacionar tempo de exposição aos raios UV com a presença ou não de lesão labial, observou-se que tanto o tempo de exposição em horas diárias $(p<0.0001)$ quanto a quantidade de dias da semana os trabalhados $(p=0.043)$ apresentavam significância estatística com a presença da lesão, o que deixa claro que, quanto maior o tempo de exposição, maior o risco de apresentar uma lesão. Já analisando as medidas de fotoproteção, não houve significância entre o tipo de fotoproteção e a presença de lesão labial $(p=0.322)$, houve uma distribuição semelhante entre os trabalhadores que tinham lesão e os que não tinham, sendo que a maioria da amostra enquadrava se no uso do chapéu ou boné. (Tabela 1).

Quanto aos hábitos de fumar e beber, quando comparamos os trabalhadores que fumam e a presença da lesão, observa-se que o percentual daqueles que fumam e não têm lesão é de $29 \%$; já os que fumam e têm lesão $47 \%$, mostrando que há uma relação estatisticamente significante $(p=0.013)$. Quanto ao uso do álcool com a presença ou a ausência de lesão, foi observada uma distribuição semelhante, onde não foi possível encontrar relação estatisticamente significativa. (Tabela 1).

$\mathrm{Na}$ Tabela 2, é possível observar o levantamento das lesões encontradas nos 62 agricultores. Queilite actínica foi a mais prevalente com $42 \%$, seguida por efélides $31 \%$, leucoplasia $9 \%$, eritroplasia $8 \%$, ceratose solar $4 \%$, herpes $4 \%$ e outras lesões que não estão relacionadas com exposição à radiação ultravioleta, neste caso, um fibroma, apenas em 1 paciente. 


\section{DISCUSSÃO}

A necessidade de compreender e analisar os fatores relacionados ao desenvolvimento da doença remete ao conhecimento das condições socioeconômicas, ambientais e políticas determinantes do processo saúde-doença de dada coletividade. Em vez de considerar apenas o conceito de fatores etiológicos e de risco, os quais se restringem aos aspectos biológicos e de caráter individual, os estudos devem voltar-se para as características de determinantes nas coletividades (LUCENA, 2012).

Este estudo não encontrou nenhuma associação entre o sexo e a presença de lesões, isto se deve principalmente porque encontramos uma distribuição semelhante entre mulheres e homens na nossa amostragem. Estes resultados não corroboram outros estudos que relatam indivíduos do sexo masculino sendo os mais susceptíveis a desenvolverem lesões (BERTINE, 2010; CARVALHO, 2008; CHEIN, 2014; LEMOS, 2009; NEVILLE, 2016). Este fato pode ser explicado pelo fato de que a natureza das ocupações ao ar livre é predominantemente masculina, além disso, as mulheres têm menos probabilidade de desenvolverem a lesão devido ao uso do batom, que pode, parcialmente, proteger os lábios do sol e culturalmente procuram mais serviços de saúde que os homens.

Os trabalhadores com idade entre 46 e 60 anos representaram a maior parcela dos casos de lesões labiais. Este resultado é decorrente do efeito cumulativo à exposição solar. Com o avanço da idade, há o aumento nas taxas de incidência do câncer de lábio, já que as implicações fisiológicas decorrentes da idade consistem em condições crônicas oriundas de fatores extrínsecos (LUCENA, 2012).

A literatura demonstra que a presença de lesões de pele e labiais está relacionada com o maior tempo de exposição à radiação (BIANCO, 2012; LUCENA, 2012; MARTINS-FILHO, 2011; OLIVEIRA, 2011). Do mesmo modo, no presente estudo, foi possível observar uma relação significativa da presença de lesões em agricultores que tinham maior tempo de trabalho sob exposição solar, demonstrando, de fato, que o efeito da radiação UV na queilite actínica é cumulativo. 
Considerando a importância de coletar dados dos parâmetros sociais, elencaram-se as variáveis: escolaridade e renda. Apesar de não encontrar associação estatística, a maioria encontra-se em níveis de escolaridade baixos, com ensino incompleto e baixa renda, o que justifica a inacessibilidade de adquirir meios de fotoproteção, principalmente o protetor solar labial, como também a falta de conhecimento do uso correto destes meios e possível dificuldade de acesso aos serviços de saúde, que foi um resultado evidente na nossa pesquisa. Tem-se a baixa renda, baseada na escolaridade reduzida, sendo fortemente associada com um maior risco de desenvolvimento de neoplasias labiais, pois os hábitos saudáveis, como alimentação e fotoproteção, são reflexos das condições socioeconômicas (LUCENA, 2012; MARTINS-FILHO, 2011; OLIVEIRA, 2011).

$\mathrm{Na}$ presente pesquisa, é notória a falta de conhecimento sobre medidas de foto proteção adequado para os lábios nestes trabalhadores, $2 \%$ da amostra usava protetor labial e este fato acontecia porque esses agricultores já haviam sido orientados previamente à presente pesquisa. O uso de boné ou chapéu foi o método mais utilizado pelos agricultores entrevistados. Apesar de ser o método mais acessível para a maioria, o boné ou chapéu não representa eficácia quanto à proteção da queilite actínica, já que esses meios de fotoproteção não apresentam materiais com fotoproteção comprovada e as áreas de mucosa labial não são protegidas totalmente pela sombra desses meios (MARTINS-FILHO, 2011). Em um estudo com 123 professores de educação física que trabalham com atividades aquáticas, durante no mínimo um ano, com idades entre 20 e 58 anos, utilizando-se um questionário com perguntas abertas e fechadas, observou-se que apenas 17,9\% protegiam-se contra a radiação solar e $64,2 \%$ estão diretamente expostos. O estudo concluiu dando ênfase ao risco do desenvolvimento de lesões decorrentes da exposição desprotegida à radiação solar (MARTINS-FILHO, 2011; OLIVEIRA, 2011).

A exposição solar desprotegida pode levar ao desenvolvimento das displasias epiteliais que, por sua vez, estão associadas a um aumento de aproximadamente $20 \%$ do risco para desenvolvimento de carcinoma epidermóide oral (ARAUJO, 2012; BERTINE, 2010). Do levantamento das lesões labiais neste estudo, a queilite actínica foi a mais prevalente de todas as lesões, com $42 \%$, estando de acordo com a literatura, onde, em um estudo para levantamento epidemiológico da queilite 
actínica entre trabalhadores rurais do município de Piracaia, São Paulo, por meio de questionário e observação clínica com 120 trabalhadores rurais, observou-se que $35,8 \%$ apresentavam quadro de queilite actínica e, segundo os parâmetros clínicos utilizados, 30,8\% apresentavam grau leve a moderado de alterações teciduais. Os autores afirmam a necessidade de orientação a trabalhadores rurais quanto aos malefícios da radiação solar e conscientização do uso de fotoproteção, não só aos que já apresentam as características clínicas de lesões labiais como queilite actínica, como também aos pertencentes aos grupos de risco (BRADFORD, 2009; LUCENA, 2012).

A queilite actínica é dotada de caráter crônico, considerada pela Organização Mundial da Saúde como uma lesão potencialmente maligna, comum em indivíduos com exposição frequente à radiação solar, afetando principalmente o lábio inferior em pessoas acima dos 40 anos, do sexo masculino e leucoderma (ARAÚJO, 2012). Sua progressão lenta leva os portadores desta lesão a acreditarem que seu aspecto clínico é referente ao processo natural do envelhecimento, ignorando sua natureza evolutiva e cancerígena (BERTINI, 2010).

A maior parte da amostra constituiu-se por trabalhadores feodermas e eles representaram boa parte dos agricultores sem lesão, já os trabalhadores melanodermas representavam uma maior prevalência das lesões em geral no lábio e este dado foi estatisticamente significante. Este resultado entra em desacordo com a literatura que relata que os indivíduos com cor de pele mais escura apresentam uma maior atividade melanocítica, pois há elevada produção e dispersão de melanina, pigmento que fornece cor à pele e a protege da radiação (BRADFORD, 2009; LUCENA, 2012).

Embora a radiação UV seja, inquestionavelmente, o fator etiológico principal da queilite actínica, a malignização da lesão pode ser acentuada quando esse fator está associado aos hábitos do tabagismo e etilismo, principalmente nos fumantes de cigarros, pois o calor gerado pela queima do tabaco é transferido para a mucosa labial, como também a absorção de seus componentes tóxicos (LUCENA, 2012; MARTINS-FILHO, 2011; OLIVEIRA, 2011).

$\mathrm{Na}$ presente pesquisa, apesar de a maioria dos trabalhadores ser nãotabagista e não-etilista, foi possível evidenciar uma relação positiva significativa com 
a presença da lesão e tabagismo, mostrando que o tabaco pode influenciar o acometimento da lesão. Além disso, as pessoas que consomem álcool ou cigarros são mais despreocupadas quanto à manutenção de bons hábitos de saúde (LUCENA, 2012).

Estudos epidemiológicos contribuem para um melhor conhecimento, pelos profissionais de saúde, sobre os aspectos clínicos e fatores associados à ocorrência de lesões potencialmente malignas, pois é crescente a incidência dessas enfermidades no mundo.

Tal fato consiste em um problema de saúde pública, posto que diagnósticos tardios e a ausência de programas de promoção à saúde levam a resultados clínicos pós-tratamento bastante desfavoráveis, implicando em prejuízos estético e funcional, além de risco de morte. Na área que estuda saúde ocupacional, esse conhecimento ainda não é usado suficientemente como ponto de partida para programas de prevenção, tampouco para as adequações ambientais de trabalho.

\section{CONCLUSÃO}

A prevalência de lesões labiais em agricultores na região do Remote interior Paraibano é alta, atingindo $64 \%$ da amostra, sendo a queilite actínica mais prevalente, o que é preocupante, pois a queilite actínica é uma lesão potencialmente maligna. O que nos faz sugerir que as medidas de fotoproteção facial com o uso boné apenas não são eficientes para prevenir lesões labiais, além disso, o risco de desenvolvimento das lesões labiais está associado a faixas etárias mais avançadas, ao maior tempo de exposição à radiação ultravioleta no trabalho e a hábitos nocivos como fumar.

Por fim, é possível observar que a população estudada é bastante carente em conhecimento sobre a importância de se proteger quanto a medidas de prevenção das lesões labiais. Diante do quadro, fica claro que medidas de educação em saúde são imprescindíveis para esta população. 


\section{REFERÊNCIAS BIBLIOGRÁFICAS}

ARAÚJO, C.P, VIDAL, M.T.A, GUERGE, C.A.L, RAMOS, A.G, JÚNIOR A.A.B, RAMALHO, L.M.P SANTOS, J.N. Queilite actínica: um estudo de 35 casos com destaque para os aspectos morfológicos. RPG Rev Pós Grad, v. 19, n. 1, p. 21-7, 2012.

AUBIN F. Mechanisms involved in ultraviolet light-induced immunosuppression. Eur J Dermatl, v. 13, n. 6, p. 515-523, 2013.

BEZERRA S.M.F.N.C, SOTTO M.N, ORII N.M, CLEITON A, DUARTE A.J.S. Efeitos da radiação solar crônica prolongada sobre o sistema imunológico de pescadores profissionais em Recife (PE), Brasil. An Bras DermatoL, v. 86, n.2, p. 222-33, 2011.

BERTINI F, SGARBI, F.C, BRANDÃO, A.A.H, CAVALCANTE, A.S.R. Análisis del perfil clínico del paciente portador de queilitis actínica: importancia para el diagnóstico. Acta Odontológica, Venezuelana, v. 18, n. 3, p. 6-1, 2010.

BIANCO, B.C. Perfil clínico dos pacientes com diagnóstico histopatológico de queilite actínica e carcinoma epidermóide de lábio atendidos entre 1998 e 2009 na Universidade Federal de Santa Catarina.Trabalho para a conclusão do curso de graduação em odontologia-Universidade Federal de Santa Catarina. Florianópolis-SC, 2012.

BRADFORD PT. Skin Cancer in Skin of Color. Dermatol Nurs Associação, v 21, n. 4, p. 170$178,2009$.

CARVALHO, G.A.O, SOUZA, J.R, DE CÂMARA, J.V.F, RIBEIRO, A. DE O.P, PIEROTE, J.J.A, Aspectos clínicos, histopatológicos e tratamento de pacientes com diagnóstico de queilite actínica: revisão da literatura. Research, Society and Development, v. 9, n. 7, p. 375-385, 2020.

CAVALCANTE, A.S.R, ANBINDER, A.L, CARVALHO, Y.R. Actinic Cheilitis Clinical and Histological Features. Journal of Oral and Maxillofacial Surgery, v. 66, n. 3, p. 498-503, 2008.

CINTRA, J.S, TORRES, S.C.M, SILVA, M.B.F, JÚNIOR, L.R.C.M, FILHO, J.P.S, JUNQUEIRA, J.L.C. Queilite actínica: estudo epidemiológico entre trabalhadores rurais do município de Piracaia - SP. Rev da Associação Paulista de Cirurgiões Dentistas, v. 67, n. 2, p. 118-121, 2013.

CHEIN, H, WENG, Q.Y, FISHER, D.E. UV signaling pathways within the skin. The Journal of investigative dermatology, v. 134, n. 8, p. 2080-2085, 2014.

DAVIS, K.J, KOKKINIDES, V.E, WEINSTOCK, M.A, O'CONNELL, M.C, WINGO, P.A. Summer sunburn and sun exposure among US youths ages 11 to 18: national prevalence and associated factors. Rev Pediatrics, v. 1, n.1, p. 27-35, 2002.

LEMOS, M. A, MACIEL, G.A.O, MATOS, F.R, AMORIM, G.A, GALVÃO, H.C. Queilite Actínica: Estudo Clinicopatológico de 46 casos.[Tese de Mestrado em Patologia Oral]- Universidade Federal do Rio Grande do Norte.Centro Ciências Biológicas e da Saúde, v. 11, n. 3, p. 9-12, 2009.

LUCENA, E.E.S, BARBOSA, D.C, SILVEIRA, E, LIMA, K. Prevalência de lesões labiais em trabalhadores de praia e fatores associados. Rev Saúde Púb, v. 46, n. 6, p. 1051-1057, 2012.

LUCENA, E.E, COSTA, D.C, SILVEIRA, E.J, LIMA, K.C. Prevalence and factors associated to 
actinic cheilitis in beach workers. Oral Diseases. Rev Saúde Púb, v.18, n. 6, p. 575-579, 2012.

MARTINS-FILHO, P.R, DA SILVA, L.C, PIVA, M.R. The prevalence of actinic cheilitis in farmers in a semi-arid northeastern region of Brazil. Int J Dermatol, v.50, n. 9, p. 1109-1113, 2011.

MIRANDA, A.M.O, FERRARI, T.M, CALANDRO, T.L.L. Queilite actínica: aspectos clínicos e prevalência encontrados em uma população rural do interior do Brasil. Rev de Pesquisa em Saúde, v.1, n.14, p. 67-72, 2011.

MINISTÉRIO DA SAÚDE. Dermatoses Ocupacionais - Brasília, DF: O Ministério, 2016. Disponivel em: http://bvsms.saude.gov.br/bvs/publicacoes/protocolo_dermatoses.pdf. Acessado em: 04 de nov de 2020.

NEVILLE, B.W. et al. Patologia oral e maxilofacial. 4. ed. Rio de Janeiro: Elsevier, 2016.

OLIVEIRA, L.M.C, PALMA, A. Hábitos relacionados à exposição solar dos professores de Educação Física que trabalham com atividades aquáticas. An Bras Dermatol, v. 86, n. 3, p. 445-450, 2011.

RIBEIRO, O, DA SILVA, L.C, MARTINS-FILHO, P.R. Prevalence of and risk factors for actinic cheilitis in Brazilian fishermen and women. Int J Dermatol, v. 53, n. 0, p. 1370-1376, 2014.

SILVA, A.A. Medidas de radiação solar ultravioleta em Belo Horizonte e saúde pública. Rev Bras de Geof, v. 26, n. 4, p. 417-425, 2008. 